\section{La tutela estatal de la madre y el niño en la Argentina: estructuras administrativas, legislación y cuadros técnicos (1936-1955)}

\section{Government tutelage of mothers and children in Argentina: administrative structures, law, and technical staff (1936-1955)}

BIERNAT, Carolina; RAMACCIOTTI, Karina. La tutela estatal de la madre y el niño en la Argentina: estructuras administrativas, legislación y cuadros técnicos (1936-1955). História, Ciências, Saúde - Manguinhos, Rio de Janeiro, v.15, n.2, p.331-351, abr.-jun. 2008.

\section{Resumen}

Describe y analiza uno de los proyectos políticos que cobró fuerza en los años de entreguerras en la Argentina y tuvo permanencia durante el peronismo: la organización de la tutela estatal de la madre y el niño. Examinar la Dirección de Maternidad e Infancia permite identificar cómo esta repartición consideró al 'binomio madre e hijo', qué propuestas realizó para resolver los problemas de mortalidad infantil, qué tipo de cuadros técnicos puso a su cargo y qué limitaciones tuvo en el proceso de implementación. Asimismo, se describen los cambios y permanencias de dicha institución durante el peronismo.

Palabras clave: legislación sanitaria; cuadros técnicos; peronismo; Dirección de Maternidad e Infancia; Argentina.

\section{Abstract}

The article describes and analyzes one of the political projects that gained strength in Argentina during the between-war years and remained in place throughout Peronism: government tutelage of mothers and children. It examines how the Dirección de Maternidad Infancia viewed the motherchild dyad, how this office proposed to address the issue of infant mortality, what type of technical staff was in place, and what limitations were encountered in trying to enforce these ideas. It also looks at what changed and what stayed the same at the office under Peronism.

Keywords: sanitary legislation; technical staff; Peronism; Dirección de Maternidad e Infancia; Argentina. 
$\mathrm{L}$ a necesidad de crear leyes e instituciones para proteger a las madres y a sus hijos aparece en las discusiones de la esfera pública argentina, en consonancia con la de la mayor parte de los países occidentales, en las últimas décadas del siglo XIX. Su objetivo principal se centra en la lucha contra la incidencia negativa de la mortalidad infantil en el crecimiento de la población local. De todos modos puede ser pensada, también, como parte de una agenda política reformista que brega por propósitos más amplios como el reordenamiento social, la mejora de las condiciones de vida de los sectores populares y la vigilancia y moralización de la población (Suriano, 2004).

Así, para combatir la mortalidad infantil, junto con los esfuerzos de instituciones públicas, pero principalmente de las privadas, por educar a las madres en los conceptos básicos del cuidado, higiene y alimentación de sus hijos recién nacidos, se propone corregir y, en todo caso penalizar, prácticas sociales tan extendidas como el infanticidio, el abandono de niños y la 'ilegitimidad' conyugal. Con todo, las medidas más eficaces para revertir este problema demográfico se centran en torno a las mejoras de las condiciones sanitarias y de vida a fin de evitar las principales causas de defunciones infantiles: las enfermedades infectocontagiosas, la diarrea y la enteritis (Mazzeo, 1993; Celton, 1995; Mead, 2000; Nari, 2004, p.24-28).

Durante la primera posguerra, la protección de las madres y de sus hijos se enmarca en un conjunto de problemas que le aportan un inusitado protagonismo y dinamismo político. En primer lugar, la declinación del flujo inmigratorio como consecuencia del conflicto bélico, las políticas restrictivas del Estado argentino y la crisis económica debilitan la confianza poblacionista en la inmigración ultramarina como multiplicadora de la población local. Por otro lado, el análisis de los resultados del censo de población de 1914 muestra la disminución del número de nacimientos, principalmente en los grandes centros urbanos. Como resultado comienza a pensarse en la necesidad de reforzar los factores endógenos que aseguren el incremento demográfico (Biernat, 2005a, p.217-245). Así todo, el ascendiente de estas ideas en la reflexión argentina responde a la fuerte impronta del 'natalismo' en las concepciones y políticas demográficas francesas, desde las primeras décadas del siglo XX, e italianas, durante la entreguerras (Reggiani, 1995-1996; Ipsen, 1997).

Un segundo aspecto se vincula con el fortalecimiento del discurso eugenésico en los ámbitos académicos, políticos e institucionales. Esta corriente de pensamiento, partiendo de la premisa de que todos los caracteres de los seres humanos son hereditarios, tanto las capacidades y talentos como la propensión a la enfermedad, se propone lograr el mejoramiento de la 'raza' blanca a través de la reproducción de determinados individuos o grupos humanos calificados como 'mejores', inhibiendo la multiplicación de otras personas consideradas 'inferiores' o 'indeseables'. En la Argentina, la eugenesia se interpreta en clave 'neolamarckiana'. Según esta visión, las características morfológicas y funcionales de los individuos pueden ser modificadas por el medio social y ambiental, transmitiéndose por vía hereditaria y pasando a formar parte de su acervo genético (Stepan, 1991; Miranda, Vallejo, 2005; Biernat, 2005b). A partir de la generalización de esta doctrina, compartida por defensores de las más variadas corrientes ideológicas, el binomio madre-hijo cobra importancia en tanto principal responsable del futuro de la raza y, por ende, del progreso de la Nación. 
Un tercer nivel de discusiones está asociado al impacto que los cambios sociales producen en el universo de certezas morales. La creciente incorporación de las mujeres al mercado de trabajo, sobre todo de aquellas que pertenecen a los sectores populares, es interpretada por los contemporáneos, como una traición a sus 'deberes maternales' y como un riesgo para su 'virtud'. De allí la necesidad de reorientarlas para que abandonen sus responsabilidades laborales y regresen a su hogares y, en caso de no ser posible, protegerlas de las amenazas que pueden sufrir su potencial reproductivo y sus funciones maternales a través de una serie de leyes como la prohibición de su desempeño en empleos considerados insalubres y del despido por embarazo, la licencia remunerada por maternidad antes y después del parto, el permiso para amamantar y la instalación de salas cuna en los lugares de trabajo. Estas medidas se complementan, desde lo ideológico, con el proceso de naturalización de la maternidad, intentando concientizar a las mujeres de su carácter instintivo y del binomio madre-hijo, explicando una relación social por sus caracteres biológicos (Nari, 2004; Ramacciotti, 2005b).

Un cuarto nivel de discusión se centra en la necesidad de establecer, a nivel nacional, un sistema sanitario encargado de la protección de la madre y el niño. Como en tantas otras áreas de la atención de la salud y la acción social, los distintos niveles de gobierno y las esferas pública y privada se superponen, combinando diferentes grados de autonomía y dando como resultado un servicio ineficiente. El Departamento Nacional de Higiene es visualizado como escenario privilegiado para la organización de una estructura administrativa que asegure la centralización de la protección del binomio madre-hijo. Este desafío parece responder a un proyecto político mucho más ambicioso de un grupo de médicos quienes, teniendo como epicentro esta repartición nacional, intentan dar respuesta a los problemas sociales desde el ámbito de la higiene y colonizar la estructura burocrática del Estado en su calidad de expertos. Si bien este proceso se había iniciado en las últimas décadas del siglo XIX, en los años 1930 de la siguiente centuria se acentúa (González Leandri, 2005). Por un lado, la creciente intervención estatal en la regulación de la vida económica y social expande su burocracia y las posibilidades de acceder a ella. Por otro parte, la demanda oficial de galenos ya no está vinculada solamente a los esporádicos brotes epidémicos, tal cual como sucedía en el período anterior, sino que se asocia al mejoramiento de la salud física y moral de la población, presente y futura. En este sentido, la protección de la madre y el niño comparte la agenda de preocupaciones oficiales con otros temas como el tratamiento de la tuberculosis o la profilaxis de las enfermedades venéreas (Armus, 2004; Biernat, 2007).

De todos modos, resulta indispensable un ordenamiento legal que dote a esta repartición de capacidades institucionales y recursos materiales. Para ello es necesario apelar a la acción parlamentaria, espacio en el que se hace evidente la rivalidad de intereses y proyectos en torno a la organización de un sistema nacional que involucra a funcionarios, especialistas, políticos y numerosos grupos de la sociedad civil.

Un último aspecto considerado en los debates es aquel del reclamo de reconocimiento oficial de los médicos especialistas en la protección y atención de la madre y sus hijos. La puericultura, la homnicultura, la obstetricia y la pediatría, copiando modelos de otras latitudes, surgen como las disciplinas específicas para dar respuesta a este nuevo sujeto de la medicina 
y la asistencia social (Billorou, 2006). Paralelamente reclaman el reconocimiento oficial de estas disciplinas como especialidades de la medicina y el acceso a los cargos públicos a través de concursos probatorios de idoneidad y de experiencia previa del candidato en la materia.

Las ciencias sociales en la Argentina han abordado esta temática desde explicaciones de corte cuantitativo y cualitativo. Si las primeras se enfocan en el estudio estadístico de la variación de los índices de natalidad, de mortalidad y de nupcialidad como reflejo del proceso de "transición demográfica" (Otero, 2004; Pantelides, 1983; Torrado, 2003, p.8296), las segundas orientan su análisis al plano de las ideas y debates en torno a la población deseada, a las propuestas para modificar los comportamientos demográficos entre finales del siglo XIX y los años 1940, a los enunciados legales que intentan dar respuesta a estos comportamientos y a su relación con la construcción del estado social en la Argentina (Barrancos, 2002; Di Liscia et al., 2000; Lobato, 2000; Nari, 2004).

A pesar de que muchos de estos trabajos introducen perspectivas renovadoras, notamos una carencia de información acerca de cómo estas ideas se plasmaron en políticas públicas y el grado de concreciones materiales. Para ello, analizaremos la creación de una repartición nacional en la órbita del Departamento Nacional de Higiene encargada de la tutela de la madre y el niño: la Dirección de Maternidad e Infancia a lo largo de 19 años. Estudiaremos cómo esta dependencia consideró al binomio madre-hijo, qué propuestas realizó, qué instituciones creó y qué perfil de funcionarios puso a su cargo para resolver los problemas de mortalidad infantil y de descenso de la fecundidad. En segundo lugar, nos proponemos evaluar los resultados de la gestión de este organismo estatal y los límites que se impusieron al desarrollo de su administración. Por último, poner la lupa en el período 1936-1955 nos permitirá, por un lado, dar cuenta de las continuidades y las modificaciones en la institucionalización de la protección de la madre y el niño una vez ingresados en los años del llamado peronismo clásico. Por otro lado, nos introducirá en la comprensión de los múltiples intereses que forman parte del armado de las políticas públicas.

\section{La tutela de la madre y el niño como problema de política nacional (1936-1944)}

La necesidad de crear instituciones estatales de protección materno-infantil aparece con fuerza en el discurso médico higienista a finales del siglo XIX. Una de las razones más importantes que sustentan esta demanda es la constatación de altas tasas de mortalidad infantil nacionales. La municipalidad de la ciudad de Buenos Aires es una de las primeras jurisdicciones en incluir en su agenda esta problemática. En la última década del siglo XIX la Asistencia Pública crea el Patronato de la Infancia. Esta institución filantrópica sostiene sus establecimientos - entre ellos un consultorio médico gratuito para la primera infancia, una sala cuna para menores de seis años y un internado para niños abandonados - con subsidios de la comuna y con donaciones privadas. No obstante la acción del Patronato, las autoridades de la ciudad ven la necesidad de delinear un plan de asistencia a la niñez y establecer un organismo público que se ocupe de ella. Surge así, en 1908, la Dirección de la Primera Infancia con la triple función de brindar asistencia médica, educativa y social a través de sus dispensarios de lactantes, de los institutos de puericultura y de los centros sanitarios de internación. 
El éxito obtenido por la capital federal en la disminución de sus índices de mortalidad infantil (Di Liscia, jul.-ago. 2002), la permanencia de los mismos en un nivel alto en el interior del país, la preocupación por la abrupta caída de la natalidad, fundamentalmente en los centros urbanos, y la influencia de la legislación internacional respecto de los derechos de la niñez, unidos todavía a los de las progenitoras, se constituyen en razones de peso para reclamar la organización, a nivel nacional, de la tutela de la madre y el niño.

El Departamento Nacional de Higiene brinda una respuesta oficial creando, en 1923, la Sección de Asistencia y Protección a la Maternidad y la Infancia. Su director, Gregorio Aráoz Alfaro, reconocido pediatra e higienista, justifica esta determinación en la necesidad de revertir las "cifras realmente pavorosas" de mortalidad infantil registradas en algunas provincias y territorios nacionales, atribuyendo sus causas a la "falta de asistencia médica y social a la madre y el niño y a la ignorancia de las madres de la población en general sobre higiene infantil y puericultura". Para subsanar estas carencias propone la instalación de consultorios, dispensarios de lactancia, centros de atención materno-infantiles (fundamentalmente en aquellas regiones donde la mortalidad infantil es elevada) y la organización de un sistema de visitadoras de higiene social (Aráoz Alfaro, 1928). No obstante la insistencia de Aráoz Alfaro de contar con un presupuesto exclusivo para la División de Maternidad e Infancia, dicha repartición lleva a cabo su labor con una exigua cifra anual (Olarán Chans, Siri, 1929).

Paralelamente a estas iniciativas, se presentan en el Congreso Nacional varios proyectos legislativos. En mayo de 1926, Aníbal Olarán Chans y Luis Siri, responsables de la División de Maternidad e Infancia, hacen público en la Sociedad Argentina de Pediatría un programa de asistencia y protección de la madre y el niño. Dicho proyecto es remitido al Congreso de la Nación donde, después de casi diez años, es presentado por el senador Alfredo Palacios a la Comisión de Legislación. Finalmente, el 21 de diciembre de 1936 se sanciona la ley 12.341 con la que se crea la Dirección de Maternidad e Infancia, bajo la Dependencia del Departamento Nacional de Higiene. Su finalidad es "propender al perfeccionamiento de las generaciones futuras por el cultivo armónico de la personalidad del niño en todos sus aspectos, combatiendo la morbimortalidad infantil en todas sus causas y amparando a la mujer en su condición de madre o futura madre". Se propone como objetivos la asistencia pre concepcional, del embarazo y el parto, la vigilancia del niño desde su nacimiento a través de fichas sanitarias individuales, la lactancia materna, la alimentación racional y la protección social de los niños necesitados. Su radio de acción incluye a todo el país, propendiendo a que cada pueblo o ciudad posea, cuanto menos, un centro de higiene maternal e infantil. Ejerce la vigilancia sobre todas las instituciones oficiales y privadas que se ocupen de la asistencia y protección de la maternidad y de la infancia, con excepción de las que dependen de la Sociedad de Beneficencia.

Un decreto reglamentario de marzo de 1937, elaborado por Olarán Chans y Siri, determinará las divisiones en las que se organizará la Dirección y las responsabilidades de cada una de ellas: La División de Higiene Social de la Infancia, encargada de hacer investigaciones demográficas, estadísticas sanitarias y de organizar la propaganda higiénica y la educación popular sanitaria; la División de Eugenesia, Maternidad y Primera Infancia, ocupada de la creación, dirección técnica y vigilancia de las instituciones o servicios de 
atención de la madre y el niño; la División de Edad Pre-escolar, Escolar y Adolescencia, abocada a la aplicación de las medidas que se establezcan para su protección física, moral y social; la División de Infancia Abandonada, Enfermos y Anormales, prevención del abandono; la División de Inspección y Legislación, ocupada del control, técnico y administrativo de todas las instituciones, oficiales y privadas, encargadas de la asistencia y protección maternal e infantil, salvo las que dependen de la Sociedad de Beneficencia; la División de Servicio Social, encargada de la organización y contralor del servicio social en todas las instituciones de asistencia y protección maternal e infantil y la creación de la Escuela de Servicio Social de la Infancia.

De todos modos, la ley 12.341 demora dos años para ser reglamentada. Una de las razones de este retraso es un recurso que interpone el Patronato Nacional de Menores. Dicho organismo de carácter privado, dependiente del Ministerio de Justicia e Instrucción Pública, encargado de la asistencia de la infancia abandonada y delincuente, considera que la mencionada ley avanza sobre atribuciones consideradas propias. El escenario político se hace complejo.

El primero de julio de 1937 el Patronato envía una carta a su ministerio de tutela oponiéndose a la reglamentación de la ley 12.341 y amenazando con abandonar la "obra de organización y tratamiento de los menores". En primer lugar, afirma que el reglamento extralimita el alcance de la ley, la protección de la primera y la segunda infancia (hasta los siete años), superponiendo facultades conferidas al Patronato desde 1931. En segundo lugar, sostiene que "la obra de protección a la infancia es esencialmente pedagógica" y, aunque no descarta el amparo material del niño desvalido y su examen médico clínico, "toda la obra responde en su conjunto a un concepto jurídico social, siendo lógico entonces que deba depender solamente de los tribunales para menores". En conclusión, la creación de institutos médicos tiene una finalidad completamente ajena a la protección de la infancia. Por último sugiere al Departamento Nacional de Higiene que se dedique a lo que es propio a su función, en cuanto a la primera y la segunda infancia y a las enfermedades que asolan a la niñez en todo el país.

El Ministerio de Instrucción Pública y Justicia envía el reclamo al Ministerio del Interior y éste al Departamento Nacional de Higiene. Según la opinión del director esta repartición, Miguel Sussini, no existe conflicto jurisdiccional entre la Dirección de Maternidad e Infancia y el Patronato Nacional de Menores puesto que las funciones del último no son las de velar por la salud de los menores en general, sino "sobre aquellos cuyos padres tuvieran pérdida transitoria o definitiva de la patria potestad". Según Sussini, en la carta enviada por el Patronato se le confiere "un significado y una extensión abusiva al término 'menores' - la mayoría de los cuales no son delincuentes y abandonados - por cuya salud y desarrollo debe velar la Dirección de Maternidad e Infancia". Mientras que el Patronato ejerce una acción restringida de carácter "jurídico-tutelar", a la Dirección le incumbe desplegar "una acción amplia de higiene social de la infancia en el país, bajo todos sus aspectos y en particular en lo que se refiere a la eugenesia y a la mortinatalidad". Por otro lado, considera que el límite de la infancia es la pubertad, determinada por ley civil 12 años en la mujer y 14 en el varón, siendo, además, un "concepto universal, médico y social". Por último, insiste en que, según la ley 12.341, la Dirección de Maternidad e Infancia "centraliza la 
supervisión de la asistencia y protección del niño, cualquiera sea la institución que lo asista o proteja".

El procurador del tesoro se expide afirmando que el decreto reglamentario "ha excedido el espíritu de la ley 12.341" ya que de todos sus articulados surge que el contralor del Departamento Nacional de Higiene se extiende tan solo a la primera y segunda infancia. Según este funcionario, pretender extender la minoridad a los 22 años sería atentar contra el espíritu de la ley en cuyo artículo 13 se ha empleado "indebidamente la palabra 'menores', cuando la que correspondía en realidad era la de infantes". En consecuencia, sugiere que se modifique el decreto.

Por su parte, el procurador general de la Nación sostiene que hasta ese momento no se ha registrado un caso concreto de superposición de funciones pero, para prevenirla se sugiere modificar el decreto. Considera "insostenible que la nueva ley haya colocado bajo la dependencia de la Dirección de Maternidad e Infancia al Departamento Nacional de Trabajo, al Consejo Nacional de Educación, al Patronato aludido, y a todos los internados, escuelas, seminarios y asilos, cárceles y establecimientos diversos donde se imparta enseñanza o cuidados a menores de edad". Como la tutela de los menores que ejerce el Patronato es encargada directamente por los jueces, éste depende del Ministerio de Justicia. En conclusión "no se advierte cómo el Departamento Nacional de Higiene pudiera tomar a su cargo la dirección de un asunto que, si bajo algún aspecto atañe a la salud, como todos, en lo fundamental comporta al ejercicio de una prerrogativa judicial".

La resolución a este conflicto de superposición de atribuciones está dada por el decreto 5.520, del 15 de junio de 1938 que establece una nueva reglamentación de la ley Palacios. Se crean nuevas divisiones de la Dirección y las existentes hasta el momento sufren modificaciones. La División de Eugenesia, Maternidad y Primera Infancia, pasa a ocuparse únicamente de eugenesia y maternidad; se crean las divisiones de Primera Infancia (hasta los dos años y medio) y de Segunda Infancia (edad preescolar); desaparece la División Edad Preescolar, Escolar y Adolescencia; las divisiones Infancia Abandonada-Enfermos y Anormales y la de Servicio Social, se concentran en una sola: Niños Enfermos, Anormales y Necesitados y se crea la División Odontológica (asistencia odontológica preventiva y curativa de las madres y los niños).

Unos años más tarde, los puericultores a cargo de la Dirección de Maternidad e Infancia subrayan las graves consecuencias que para la actuación de este organismo nacional ha tenido la restricción de su incumbencia a lo niños menores de seis años. Según Olarán Chans, "no podría concebirse, por ejemplo, que en el Centro de Higiene Maternal e Infantil de Río Grande (Tierra del Fuego) se interrumpiera la asistencia médica, odontológica o social de un niño por el hecho de haber cumplido seis años de edad, máxime cuando esa es la única institución médica que existe en el lugar" (Olarán Chans, 1941, p.163). Por su parte, Pilades Dezeo - jefe de la División de Higiene y Servicio Social de la Dirección de Maternidad e Infancia - advierte que la ley, limitada por el decreto de 1938, "trunca la obra en una edad de la vida del niño cuya atención médico social sigue siendo urgente y necesaria". Según su opinión, "habría bastado que el nuevo decreto reglamentario especificara que 'quedaban excluidos de la ley los niños o menores que se encuentren bajo dependencia judicial o instituciones públicas'” (Dezeo, 1939). 
Así todo, Pilades Dezeo reconoce que no se puede atacar con instituciones centralizadoras a las instituciones de beneficencia privada porque éstas son necesarias para la obra social. Instituciones autárquicas o paraestatales como el Patronato de Menores, el Patronato de la Infancia, la Sociedad de Beneficencia, la Comisión de Asilos y Hospitales Regionales, el Consejo Nacional de Educación, "cuyo desenvolvimiento es muy amplio, con muchos años de existencia y mucha gravitación económica", de sentirse atacadas "plantearían conflicto de preeminencia y desviarían a la incipiente organización de maternidad e infancia" (Dezeo, 1939, p.526).

Estas superposiciones jurisdiccionales no se limitan solamente al ámbito público y privado sino que abarcan los distintos niveles de gobierno. En efecto, muchas provincias y municipios poseen sus propios organismos de tutela de la madre y el niño, llevan a cabo su acción en forma autónoma e impiden a la Dirección Nacional que supervise su labor. Tal es el caso de Dirección de Infancia de la ciudad de Buenos Aires, de la Dirección Provincial de Tucumán o de la Caja Municipal de Asistencia Social a la Maternidad e Infancia de Godoy Cruz (Mendoza), contando las dos últimas con un aporte substancial de los sectores productivos de la región, industria del azúcar y del vino respectivamente (Argentina, 1941; Giménez Lascano, 1941; Maurín Navarro, 1943).

Como hemos visto, la Dirección de Maternidad e Infancia organiza su labor a través de centros materno infantiles dispersos en todo el territorio del país. La organización de estos centros varía de acuerdo a las necesidades y a los recursos de que dispone la Dirección. Hacia 1939 se cuenta con cincuenta en todo el país, de uno a cuatro en cada territorio o provincia. Paralelamente se construyen tres equipos para consultorios ambulantes de puericultura destinados para Salta; San Luis y Santa Rosa (Olarán Chans, 1939).

De todos modos, son muchas las dificultades con las que se encuentra la Dirección. En primer lugar, la escasez de presupuesto. Según Siri, subdirector de la repartición, en 1937 se otorgan 938 mil pesos; en 1938 esa suma es reducida; en 1939 se disminuye a ochocientos mil pesos a los que se le suman setecientos mil pesos que el Congreso destina para la creación de centros de higiene maternal e infantil y para la ampliación de servicios en regiones de elevada mortalidad infantil. Si consideramos, según lo expone Olarán Chans, que formar un centro materno infantil cuesta 22 mil pesos y si está equipado con maternidad, cien mil pesos, es poco lo que se puede lograr. En segundo lugar, existen dificultades para encontrar locales adecuados en el interior del país e imposibilidad de hacer reformas en los locales alquilados. Por último, los sueldos exiguos, la falta de idoneidad y preparación especial de médicos, enfermeras-visitadoras y preparadoras de alimentos, la necesidad de capacitar al personal (salvo al médico y a las parteras) en el Instituto Modelo de Buenos Aires a fin de uniformar las normas de todas las instituciones de la Dirección contribuyen a reconstruir un panorama bastante pesimista (Siri, 1941, p.212; Olarán Chans, 1939, p.140-142).

Vista desde el interior del país, la labor de la Dirección es aún más reducida. Víctor Frigieri, médico rural de Bernardo de Irigoyen, provincia de Santa Fe, presenta su experiencia en el $2^{\circ}$ Congreso Argentino Sanitario de Medicina Social, realizado en julio de 1942. Para Frigieri (1944) la legislación maternalista "ha sido teóricamente frondosa", pero sus resultados "prácticamente no existen". La "protección a la niñez y a las grávidas se efectúa limitadamente en zonas 'elegidas' como son las grandes ciudades, como si los niños de la 
campaña no fueran niños o no tuvieran problemas". En consecuencia, la mortalidad infantil adquiere caracteres impresionantes en el campo. En una segunda parte del trabajo, Frigieri pasa a describir la asistencia sanitaria en su departamento. En lo que se refiere a la atención hospitalaria, se cuenta solamente con dos policlínicos, por lo que madres, lactantes y niños de segunda infancia deben concurrir al consultorio externo. Una vez curados de su proceso momentáneo, no hay ningún organismo que cuide de ellos puesto que no hay maternidades en el departamento.

Por su parte Juan Maurín Navarro (1943), médico fundador de la Caja Municipal de Asistencia Social a la Maternidad e Infancia de Godoy Cruz (Mendoza), se lamenta de que la Dirección no cuente "entre los centros que constituyen su plantel actual, sino con cuatro ó cinco que tengan la aptitud funcional del de San Juan y que estén animados por análogo espíritu de trabajo y de dedicación. El resto ha sido sojuzgado por el electoralismo que lo degenera todo y que ha hecho que los contados organismos que debían ser baluartes de la salud del niño en el interior se conviertan en menguados refugios de la compincharía electoral".

Por último Roberto Bilella (1941), médico de San Juan, analiza los altos índices de mortalidad infantil en su provincia, 33\% a 35\% (sic) en los años 1936, 1937, 1938, y sitúa en cuarto lugar, entre las causas de decesos de lactantes, a la falta de asistencia sanitaria. Según su descripción, no existe en San Juan una organización que se ocupe de la atención del niño; se cuenta con un solo Centro de Protección Materno Infantil de orden nacional, "modelo por su organización y con un pequeño internado"; no hay salas para internar lactantes; el número de camas para maternidad es de cuarenta para una población de 210 mil habitantes; en los departamentos no existe ninguna organización de protección materno-infantil; los lactantes deben ser llevados a los consultorios externos de la Asistencia Pública de la ciudad capital, atendidos por dos médicos, donde concurren 150 niños diariamente, y no hay una ordenanza apropiada sobre extracción, envase y expendio de leche de vaca.

En 1940 Luis Siri (1941) propone como solución a la incapacidad de la Dirección de Maternidad e Infancia de desplegar su acción efectiva en el extenso mapa argentino la adopción de un plan nacional de higiene, asistencia y previsión social. En su opinión, la principal falla de los planes de organización de la asistencia social es que buscan la solución "partiendo de los aspectos formales alcanzados por las múltiples instituciones en que se divide la atención de la salud, en vez de ir a buscar sus fundamentos en las raíces mismas de la realidad del país". Por otro lado, remarca el escaso valor de las estadísticas de demografía, como elemento de juicio en el estudio de los problemas médicos sanitarios, por realizarse éstas de forma insatisfactoria, y, por último, la falta de ideas precisas sobre la forma en que se ha de efectuar la coordinación de los servicios de asistencia social.

Una vez hecho el diagnóstico, para Siri las etapas que debe seguir el plan general de la protección de la madre y el niño son la determinación de la participación que en los planes regionales ha de corresponder a los gobiernos nacional, provincial y municipal; la coordinación de las actividades de todas las instituciones o servicios, oficiales y privados que se ocupen de la madre y el niño; la armonización de las medidas legales de orden provincial o municipal con la ley 12.341; la coordinación de los organismos provinciales y 
municipales de las tareas relativas a vigilancia de instituciones, supervisión de la salud del niño, educación popular sanitaria y lucha contra el curanderismo; la creación de centros de investigación y escuelas prácticas de puericultura, de orientación regional para el estudio permanente de los problemas higiénico sociales de cada región y la formación de personal capacitado para actuar en su zona de influencia; la determinación de los recursos necesarios para la instalación y el funcionamiento de todas las instituciones comprendidas en el plan y el aporte que ha de corresponder a cada uno de los gobiernos nacional, provincial y municipal y a la obra privada.

Como primer paso de este ambicioso plan, Siri crea en 1939 el Gabinete de Geografía Social, bajo la órbita de Maternidad e Infancia, con el fin de estudiar las características y la distribución de la población en las distintas zonas del país a través de una encuesta. A pesar de sus esfuerzos por elaborar y poner en práctica un plan sanitario y de asistencia social, sus resultados parecen ser muy modestos. A los problemas de superposiciones jurisdiccionales entre distintas instituciones y niveles de gobierno, a la reticencia de las provincias a entregar información estadística a la Dirección de Maternidad e Infancia, a la falta de presupuesto y personal, se le suma la resolución del decreto 31.589, del 30 de noviembre de 1944, que excluye a la Dirección General de Salud Pública de las funciones de asistencia social y beneficencia, transfiriendo esta responsabilidad a la Secretaría de Trabajo y Previsión, y restringe la jerarquía institucional de sus organismos convirtiéndolos nuevamente en divisiones. Este recorte de sus funciones, concretado principalmente en la reducción presupuestaria y de su capacidad de asistencia social, representará para la Dirección General de Salud Pública, y para todas sus reparticiones, una ostensible limitación de su poder de intervención en las provincias y en los territorios nacionales y, en consecuencia, de su capacidad de organizar en el ámbito nacional un sistema de asistencia sanitaria efectivo.

\section{La Dirección de Maternidad e Infancia durante el primer peronismo}

A la llegada de Perón al poder se producen una serie de modificaciones institucionales. En el ámbito sanitario, el 23 de mayo de 1946 se suprime por decreto la Dirección Nacional de Salud Pública dependiente del Ministerio del Interior (1944-1946) y se crea la Secretaría de Salud Pública. El neurocirujano Ramón Carrillo ocupa el cargo de secretario y, en 1949, se convierte en el primer ministro de salud.

En un claro intento por abandonar la vinculación con tradiciones previas, muchas instituciones cambian sus denominaciones. No obstante ello, la Dirección de Maternidad e Infancia mantiene su nombre y en 1947 es designado a su cargo Jaime Moragues Bernat. Este prestigioso obstetra diseña la planificación para dicha área. Su preocupación central es la despoblación y su propuesta gira en disminuir la mortalidad infantil a los índices históricos alcanzados por la ciudad de Buenos Aires. Pero a fines del mismo año que es nombrado responsable es relevado de su cargo.

Cabe preguntarse por qué separar de sus funciones al asesor técnico que había colaborado en la planificación sanitaria y poseía una importante trayectoria académica y reemplazarlo por Lorenzo Pignone, un pediatra sin antecedentes relevantes. Resulta sugerente que dentro del área de salud pública del primer gobierno peronista, las personalidades vinculadas con 
una acción científica o de gestión durante los años previos, no fueron puestas en cargos directivos claves. No obstante ello, mantienen puestos de asesores y/o representantes del Estado ante congresos internacionales. Así por ejemplo, Moragues Bernat es nombrado asesor consultivo de la Secretaría de Salud Pública; Gregorio Aráoz Alfaro, ex presidente del Departamento Nacional de Higiene y ligado a la gestión del presidente radical Marcelo T. de Alvear, representa al gobierno ante la reunión del Consejo Directivo del Instituto Internacional Americano de Protección a la Infancia, realizada en Montevideo en 1951; Olarán Chans, director de Maternidad e Infancia entre 1937 y 1945, es enviado a Estados Unidos, en 1948, con el fin de estudiar las organizaciones médicas y científicas dedicadas a la higiene materno infantil. Esta experiencia internacional no es volcada en la gestión pública ya que a su regreso no ocupan cargos en la misma.

Pignone, sin la trayectoria académica de su antecesor, mantiene su nombramiento hasta septiembre de 1949, momento que se hace cargo de dicha repartición el pediatra Pedro Alberto Basílico. Esta rotación de cuadros técnicos parece estar en consonancia con el recorrido de varios de los técnicos de la Secretaría y del Ministerio de Salud Pública, a excepción de la larga gestión de Ramón Carrillo quien ocupó su cargo por ocho años. Los cambios de gestión son un indicador de la modificación de las cuotas de poder entre grupos y puede ser entendida como un límite hacia la profesionalización, giros en la administración y por lo tanto suponen una alta incertidumbre de las reglas de juego.

Entre fines de 1946 y 1948, no existen medidas significativas vinculadas a la protección de la madre y el niño. En efecto, los cambios de funcionarios y la adecuación de nuevos objetivos en la planificación dan lugar a un tiempo de espera en la implementación de la práctica política. La entrada de Pignone coincide con el momento de mayor dinamismo constructivo que se detiene hacia mediados de 1949 y su desplazamiento concuerda con un cambio de rumbo en la política de la Dirección de Maternidad e Infancia dado principalmente por un recorte presupuestario. De tener como eje la construcción de centros maternos infantiles pasa a tener como foco la difusión de medidas para la higiene y el cuidado de los recién nacidos.

De la maternidad integral al centro materno infantil

Dentro de los tres ejes que organizan la salud pública durante el primer peronismo - la medicina asistencial que apunta a la cura de los individuos; la medicina sanitaria o profiláctica, centrada en la erradicación de los factores medioambientales que inciden directamente en la difusión de enfermedades y la medicina social o preventiva, encargada de tomar medidas encaminadas a mejorar el ámbito económico y social - la Dirección de Maternidad e Infancia forma parte de esta última (Argentina, 1947, 1951a).

Según el Plan Analítico de Salud Pública de 1947, la Dirección de Maternidad e Infancia debe ejercer las funciones relativas a la asistencia y protección integral de la madre, de la futura madre y del niño por medio de las llamadas 'maternidades integrales'. La centralización de la asistencia sanitaria, social y moral bajo un solo organismo pretende evitar la falta de coordinación entre la iniciativa nacional, la provincial y la privada. Se propone construir fundamentalmente Jujuy, Salta y Tucumán, donde los índices de mortalidad son mayores (Argentina, 1947, p.442). 
Desde el punto de vista técnico, la maternidad integral abarca los servicios de obstetricia y ginecología, puericultura e infancia. Desde el punto de vista social, se plantea dar asilo a las embarazadas pobres, así como a las madres y sus hijos. También permitiría resolver cuestiones morales dado que se considera que la circulación de las embarazadas o parturientas en hospitales polivalentes puede traer inconvenientes éticos o afectivos para los otros pacientes. En forma complementaria y subsidiaria a las 164 maternidades que se planifican, se estipula la creación de 35 centros maternos infantiles. Éstos deben estar en zonas rurales, dar asistencia en el período prenatal y post natal y su personal sería rotativo. Según lo planeado, los casos graves tienen que ser derivados a una maternidad. En franca oposición a la práctica desarrollada por el Departamento Nacional de Higiene, se considera que "la lucha por proteger a la madre y el niño solo con centros maternos infantiles es tan estéril como la lucha contra la tuberculosis reducida a dispensarios antituberculosos" (Argentina, 1947, p.455).

La maternidad integral encuentra su antecedente en un proyecto de asistencia a la madre y al niño presentado por Alberto Peralta Ramos, Ángel Sosa y Sánchez, Pedro Elizalde, Juan Garrahan, Alberto Peralta Ramos (hijo), Jaime Moragues Bernat y María Zurano en la Conferencia Nacional de Asistencia Social de 1933. En este plan la maternidad, que debería contar con personal especializado, es concebida como un concepto integral de asistencia médica y social, de enseñanza y divulgación de principios de higiene para la madre y el recién nacido. En línea con las ideas del puericultor francés Adolphe Pinard, formulan la creación de 'maternidades refugios' (Beruti, 1943, p.548-557).

Es indudable que la propuesta de brindar asistencia médica, social y moral para la madre y el niño, plasmada en la legislación en 1947, es tributaria de los proyectos de los años 1930. No obstante ello, estos planes no cuajan en la práctica política y esto echa luz acerca de las distancias entre la enunciación del programa de gobierno y el campo de las concreciones materiales. La distancia entre el ideal y la práctica no debe ser interpretada como un fracaso de la política. Entendemos que hay que profundizar tanto en las demandas de diferentes actores sociales y políticos que pudieron haber incidido no sólo en los cambios de funcionarios sino, también, en la introducción de modificaciones en el rumbo de las políticas y en el recambio de los cuadros técnicos. Así mismo, los efectos que puede generar la implementación (o la ausencia) de una política también producen modificaciones sobre las agendas públicas que deben ser consideradas en el análisis. Rescatar este aspecto nos permite visualizar el proceso de construcción que lleva implícita toda política social (Ramacciotti, 2006).

Entre fines de 1947 y 1952 se habilitan más de cincuenta centros maternales en diferentes partes del país, pero no se crean nuevas maternidades. La estrategia para dar solución a las problemáticas sanitarias de la madre y el niño se hacen desde el nivel administrativo local acompañado del asesoramiento técnico y normativo otorgado por la Dirección de Maternidad e Infancia (Menchaca, 1953). En efecto, los centros maternos infantiles, idealmente pensados como eslabones de un sistema más amplio, son en la práctica depositarios de un gran margen de autonomía. Poseen personal rotativo lo cual abre la posibilidad de que, en ciertas oportunidades, se queden sin médicos o sin personal especializado. Lorenzo García (1954, p.63), fiel colaborador de Carrillo durante la etapa 
del Ministerio de Salud, sostiene que los objetivos del primer plan quinquenal se retardan debido a la carencia de enfermeras especializadas, de médicos pediatras, de puericultores y de visitadoras de higiene.

En 1950 el Ministerio de Salud Pública, en función de ajustar las atribuciones y jurisdicciones a lo realmente existente, reorganiza su reglamento interno y en esta oportunidad no aparece la maternidad integral. Siguiendo esta línea, el Plan Esquemático de Salud Pública (1952-1958) estipula que el "Centro de Salud Materno Infantil es el mejor instrumento de lucha contra la mortalidad infantil" y agrega que el capítulo escrito por Moragues Bernat, está plagado de "planteos vacilantes y poco actualizados" y su fracaso se debe, en gran medida, por no haber otorgado importancia a los centros materno-infantiles. En un claro sentido reparador se postula que estos centros se deben instalar "como un anexo a los hospitales existentes y sólo construirlos en los lugares donde no existan hospitales" (Argentina, 1950, 1951b).

A partir de 1947, la Dirección de Maternidad e Infancia controla los hospitales que cuentan con maternidad y que anteriormente dependían de la Sociedad de Beneficencia de la Capital Federal. Esta transferencia no está libre de escollos ya que uno de los tópicos que se discute en la Cámara de Diputados al crearse la Dirección Nacional de Asistencia Social es decidir qué organismo tendrá el control de los hospitales que anteriormente dependían de la Sociedad de Beneficencia. La disputa está centrada sobre quiénes tendrán mayores atribuciones para inspeccionar estos hospitales. Los 'médicos' de la Secretaría de Salud, en tanto figuras técnicas y poseedores de un conocimiento específico, o la Secretaría de Trabajo y Previsión, en función del predominio de figuras políticas. Este argumento, basado en la dicotomía 'técnicos' opuestos a los 'políticos', da cuenta de una disputa de poder y de una búsqueda de criterios de legitimidad que sustente la existencia, real o simbólica, de nuevas instituciones en el entramado estatal. Las referencias a la necesidad de "hombres prácticos" en oposición a los "hombres políticos" es un elemento recurrente en el discurso político argentino que se remonta al ideario liberal. El supuesto es que la entrada de ciertos profesionales a la arena del Estado redundaría en un beneficio para el progreso de la nación. ${ }^{8}$

Las maternidades que dependían de la Sociedad de Beneficencia de la Capital Federal, que pasan a la órbita de la Secretaría de Salud, están ubicados en Buenos Aires, lo cual indica trabas en la satisfacción de las demandas sanitarias en el interior. Muestra de estas limitaciones son los constantes reclamos sobre la necesidad de crear servicios de atención a la madre embarazada y al niño en las cartas enviadas por los particulares y las asociaciones como respuesta al pedido de iniciativas para reformular el $2^{\circ}$ Plan Quinquenal en diciembre de 1951. A modo de ejemplo, podemos mencionar el pedido de la Unión de Mujeres Argentinas de Liniers, quienes solicitan una maternidad en la zona ya que las madres deben acudir al Hospital Salaberry y este nosocomio no cuenta con camas suficientes. Por su parte, Armando Berraguti solicita la construcción de un hospital provincial en Santa Fe ya que el existente carece de sala de maternidad y de sala de niños. Agrega que, dada la existencia de escasos servicios e insumos, los trabajadores deben concurrir a los sanatorios privados. ${ }^{9}$

La pregunta es por que el ideal de brindar "ayuda médica y social integral" está limitado en la práctica política. Por cierto, la planificación sanitaria debe ser reformulada, dadas las 
disputas jurisdiccionales, a partir de la creación de la Dirección de Asistencia Social bajo la tutela de la Secretaría de Trabajo y Previsión. Esta dependencia, dirigida por Armando Méndez San Martín, tiene por función dar protección a la madre desamparada debiendo concurrirse por todos los medios a evitar la disolución del binomio madre-hijo. Así mismo, la asistencia a mujeres pobres que comienza a brindar Eva Perón es otro elemento que limita la planificación sanitaria. Muestra de ello es que a mediados de 1947, después de ser intervenida la Sociedad de Beneficencia, es el Estado quien resguarda a las mujeres con o sin hijos. Una vez creada, en el año 1948, la Fundación de Ayuda Social María Eva Duarte de Perón, este hogar de tránsito depende de esta institución. Además se construyen dos hogares de tránsito más y el hogar de la empleada General José de San Martín. A estas instituciones de corte asistencial-médico debe sumarse la construcción de los policlínicos de la Fundación Eva Perón.

En un primer momento parecieran existir relaciones y lógicas comunes entre la Secretaría de Salud Pública y la Fundación Eva Perón. En función de dar cuenta de las demandas sanitarias y sociales que provienen del interior del país, la Secretaría de Salud Pública crea, a principios de 1947, un Servicio de Ayuda Médica Integral. Convertida en dirección en 1950, esta repartición se aboca exclusivamente a dar satisfacción a los pedidos de personal, remedios y servicios médicos por parte de la Fundación Eva Perón. Esta descripción da cuenta de la existencia de áreas de competencias compartidas entre la fundación y la secretaría. Esta relación complementaria se diluye entrados los años 1950. Por un lado como lo demostraremos más adelante - la Fundación Eva Perón logra mayor autonomía económica y, por otro, limita la dependencia con el Ministerio de Salud en relación a la dotación de personal técnico.

Las trabas que trae en la práctica el proceso de especialización médica es otro factor importante a la hora de pensar en los límites operativos de la maternidad integral. Francisco Menchaca, profesor adjunto de higiene y medicina preventiva de la Facultad de Medicina de la Universidad del Litoral sostiene que la excesiva diversificación de las especialidades médicas y su atención en distintos centros especializados traen como consecuencia un mal uso de los recursos. De allí que proponga su centralización en una sola institución que brinde asistencia integral a toda la familia - y no sólo a la madre y al hijo - a fin de evitar las interferencias en la atención y la duplicación de las tareas. En esta misma línea, Carrillo cree que la concentración de diferentes servicios en un solo edificio es la forma más eficaz y eficiente de resolver las múltiples demandas sanitarias. El policlínico, que abarca también los servicios de maternidad, se propone como una estrategia más ajustada ante la necesidad de centralizar servicios con miras a racionalizar el presupuesto sanitario (Menchaca, 1952; Carrillo, 1951, p.298).

\section{Los únicos privilegiados son los niños}

Desde la segunda década del siglo XX, distintas miradas ponen el acento en la necesidad de valorizar a la infancia como una etapa de la vida autónoma de la adulta y de cuyo crecimiento saludable depende el desarrollo, físico, psíquico y social de las personas. Con diferentes argumentos, el cristianismo, los organismos de derechos humanos, los militantes socialistas y comunistas y las voces de la corporación médica defienden la idea de que el 
'niño' es sujeto de derechos. En función de este clima de ideas, la Dirección de Maternidad e Infancia continúa con la línea planteada años antes de estudiar y propiciar la solución de los problemas vinculados a los niños de uno a seis años. En esta valoración de la niñez nos encontramos con un terreno menos retórico y más ligado a la acción política.

Si bien a partir de 1948 se concreta un dinamismo constructivo plasmado en la creación de centros de higiene materno-infantiles, éste se diluye cerca de 1950. Es en este momento cuando se hacen notorias las medidas vinculadas a la protección a la niñez representadas por los controles a la alimentación del recién nacido y los consejos a las madres sobre la mejor manera de brindar alimentos nutritivos a sus niños. En función de mejorar los índices de mortalidad infantil, preocupación constante de la Dirección de Maternidad e Infancia, se realizan informes sobre la incidencia del clima en este fenómeno, se reglamentan las cocinas de leche, se modifica el registro del recién nacido debiendo las autoridades hospitalarias estar en estrecha relación con las del Registro Civil a fin de establecer la correcta filiación del nuevo ser, se realizan inspecciones en las salas cunas y en los jardines de infantes, se entrega a las madres internadas un ajuar para sus bebés y cartillas con consejos sobre la higiene y sobre la evolución cronológica deseada para sus hijos. En esta valorización de la niñez se instituye la celebración de la Semana del Niño que, junto a la Semana de la Salud de Trabajador y del Día del Médico, constituyen instancias festivas en el calendario sanitario (Argentina, 1952, p.391-417).

En 1948 se crea el Consejo del Niño, bajo la órbita de la Dirección de Maternidad e Infancia y se institucionalizan los problemas que amenazaban a la niñez. En América Latina, este tipo de instituciones tuvo como antecedente a Uruguay que en 1934 organizó y puso en funcionamiento un Consejo del Niño y además sancionó un Código del Niño (Birn, 2006).

En la Argentina, el Consejo del Niño se abocará al cuidado de la primer infancia, momento considerado fundamental para asegurar el posterior desarrollo del "hombre del mañana". Se consideran a la alimentación 'racional', por medio de la instalación de lactarios, y la visita seguida al dispensario, como elementos que asegurarán el mejor desarrollo biológico de los infantes. En función de evitar las "lacras sociales del futuro" y lograr el "ideal superior simbolizado por una juventud fuerte sonriente y optimista", los cuidados maternales y médicos durante los primeros años son considerados claves (Argentina, 1948, p.40).

La lactancia materna es un tópico que atraviesa gran parte del siglo XX. Durante el peronismo es fuertemente estimulada y penalizada moralmente la madre que no la practica. No obstante ello, comienzan a darse recomendaciones para preparar de forma más higiénica la leche artificial. En 1951 se constituye el Servicio Nacional de la Leche que pretende limitar la mortalidad infantil por medio de una "distribución más lógica, racional y equitativa de la leche" ya que, según informes técnicos, el 70\% de la producción láctea es consumida en la región Buenos Aires-litoral en detrimento del resto del país (Argentina, 1952, p.123).

Esta intervención sanitaria es parte de una reacción política a los constantes reclamos de los profesionales de la salud y de las madres sobres dificultades que presentaba la leche artificial: las madres denuncian que se agriaba y se cortaba con facilidad, los pediatras denuncian trastornos digestivos debido a la falta de nutrientes esenciales en la leche de 
vaca. La escasez de la leche y su encarecimiento dadas las huelgas de tamberos y de los obreros de las industrias lecheras dificultan poder llevar a cabo muchas de las recomendaciones alimentarias sanitarias. En 1952 con un claro tono crítico, el organismo sanitario denuncia que en los lugares donde la mortalidad infantil hace estragos es necesario instalar tambos pero, como "esa solución escapa a las posibilidades del Ministerio", en los centros maternos infantiles se debe proveer leche en polvo, condensada y productos dietéticos derivados de la leche (Argentina, 1952, p.92).

Ahora bien, esta valorización de la niñez está vinculada con un discurso local e internacional que apunta en este sentido. Es interesante rescatar que para la Dirección de Maternidad e Infancia esta asociación discursiva en defensa de los derechos de los niños es un recurso que le permite incidir políticamente dado el recorte de presupuestario de 1949. Al no poder sustentar su visibilidad pública por medio de la construcción de centros maternos infantiles, mantiene algún espacio político a partir de la vinculación con otras dependencias sanitarias tales como la Dirección de Cultura Sanitaria, la asociación con los organismos internacionales y un mayor diálogo con los pediatras. Esta búsqueda de nuevas bases de legitimidad está en sintonía con un claro intento de mantener su existencia, real o simbólica, dentro del espacio estatal.

\section{Escasez de recursos}

Uno de los escollos que tuvo que enfrentar la Dirección de Maternidad e Infancia en los años 1940, es la escasez de recursos para hacer frente a sus necesidades. En el terreno de las construcciones hospitalarias, el Departamento Nacional de Higiene no posee el control de las construcciones hospitalarias. La decisión y el presupuesto sobre la edificación de centros sanitarios son del Departamento de Arquitectura Hospitalaria, que depende del Ministerio de Obras Públicas. El traspaso de esta dependencia a la órbita de la Secretaría de Salud Pública amplifica el dinamismo y la capacidad material para estudiar, proyectar, construir, conservar y ampliar todas las obras hospitalarias en curso y para aquellas planeadas para el futuro.

A esta institucionalidad formal se le agrega la entrada de nuevas partidas financieras por medio de la transferencia de una 'cuenta especial', compuesta por los recursos originados por un impuesto a la explotación de los casinos y los juegos de azar. Este subsidio es utilizado por la Secretaría de Salud Pública hasta mediados de 1949. Desde entonces, por medio de un decreto del poder ejecutivo, es cedido a la Fundación Eva Perón. Este recorte presupuestario, sumado al cambio de rumbo económico a partir de 1950, es objeto de duras críticas por parte de Carrillo. En 1950 publica un informe en el que sostiene que "mientras el volumen de gastos ha aumentado cuatro veces, el volumen de presupuesto solo se ha incrementado dos veces sin tomar en cuenta el mayor costo de vida". Agrega que el mayor rendimiento solo tiene por explicación "la mejor organización y un ajuste administrativo mayor en 1949 que en 1946" (Carrillo, 1950, p.77).

Si bien entre 1946 y 1947 son escasas las obras vinculadas a la protección de la maternidad e infancia, el panorama se modifica desde mediados de 1948. Es a partir de este momento y hasta 1950 donde existe un mayor dinamismo en materia de construcción de centros maternos infantiles. Durante este período se crean cerca de cincuenta centros a lo largo del 
país y se restauraran otros que habían sido edificados por el Departamento Nacional de Higiene. La poca especificidad sobre los servicios que realmente brindan, limita la posibilidad de analizar el impacto real en la vida de las personas. Esto es, muchos centros maternos infantiles solo cuentan con servicios odontológicos lo que limita la satisfacción de otras demandas. El recorte de presupuesto a partir de 1950 vacía las atribuciones efectivas de la Dirección de Maternidad e Infancia ya que las cuestiones relativas al binomio madre-hijo se trasladan a otro espacio de poder: la Fundación Eva Perón.

\section{Conclusiones}

En los años 1930 se constituyen nuevas agencias estatales que redefinen muchas relaciones cruciales dentro de la sociedad civil, aun aquellas que se encontraban tradicionalmente ceñidas al ámbito de lo privado. En el área de las políticas sanitarias esa intervención centralizadora se basa en los supuestos técnicos-organizativos de los médicos. Éstos, en tanto sus antiguas redes de inserción profesional y política, obtienen un acentuado protagonismo en la esfera estatal. En sus agendas técnicas-políticas, dos tópicos son recurrentes: la necesidad de dotar al Estado de una organización centralizada y racional de la asistencia médico social y la resolución de la llamada 'crisis poblacional' caracterizada por un decaimiento en el flujo de natalidad, principalmente en las zonas prósperas, y un empobrecimiento biológico de la población.

En este artículo nos centramos en la trama de relaciones que se constituye en torno a la Dirección de Maternidad de Infancia entre 1936-1955. Este recorte nos permite analizar las continuidades y las rupturas en un período que incluye un recambio de gobierno que signó la historia política y social en la Argentina: la llegada del peronismo y la ampliación de los contenidos de la ciudadanía social y política.

En el área de maternidad e infancia se produce un reconocimiento de los derechos de las madres y sus hijos antes de la llegada del peronismo. Este proceso no está libre de tensiones ya que la nueva repartición presenta un amplio arco de problemas: las disputas por lo que otras reparticiones interpretan como un recorte de atribuciones, la imposibilidad real de dar curso a los proyectos, dada la escasez de recursos y la falta de personal, la nueva distribución de poder entre grupos que esto genera y las dificultades para dar cauce a las múltiples realidades provinciales. Así por ejemplo, si bien la Dirección de Maternidad e Infancia pretende la superintendencia sobre las instituciones privadas o los organismos provinciales y municipales de tutela de la madre y el niño, su incapacidad material y su falta de legitimidad le impiden ejercerla en la mayoría de los casos.

Con la llegada del peronismo no se introducen cambios nominativos de esta repartición pública, pero se produce un recambio del personal político-técnico. La entrada a la administración de reconocidos obstetras establece modificaciones en la forma de plantear la planificación estatal. Discursivamente, el centro materno infantil es desplazado por la idea de maternidad integral. Pero este entramado de ideas, plasmadas en el Plan Analítico de Salud Pública de 1947, no cuaja en la práctica política ya que, luego de un recambio de personal caracterizado por la entrada de pediatras a la administración pública, se recurre a la construcción de centros maternos infantiles. 
La activa etapa constructiva comprendida entre 1948 y 1950 da una mayor corporeidad institucional a la protección materna e infantil e implica una transformación profunda en el campo de la salud pública en tanto busca proyectarse a todo el territorio del país. Es durante este lapso que se puede rastrear una acción política nacional dinámica que, no solo se caracteriza por construir obras, sino que posee la capacidad necesaria para reducir los efectos producidos por las trabas regionales, las superposiciones administrativas y la constante rotación de funcionarios.

Luego de una reducción del presupuesto se genera una poda de atribuciones efectivas dando lugar a una institución con entidad más simbólica que real. En función de mantener algún espacio de incidencia política, se incrementan las campañas de difusión sanitaria preventiva. La tutela de los niños comienza a dirigirse directamente a los niños en los consultorios pediátricos, en las escuelas o en las colonias de vacaciones, en función de la autonomización de sus derechos y de la penalización de las madres por las conductas 'irracionales' hacia sus hijos. Por último, los médicos parecen ser recluidos a sus saberes disciplinarios o a su función técnica y desplazados de su lugar de propulsores de un proyecto político.

Frente a este reacomodación política, la Dirección Nacional de Asistencia Social y, principalmente, la Fundación Eva Perón quedan como las principales instituciones que cubrirán las demandas sanitarias y asistenciales de las madres y sus hijos ya que son las que portan recursos simbólicos y efectivos para solucionar los problemas que se consideran prioritarios. Estas diferentes vías para satisfacer las necesidades asistenciales de los niños $y$, secundariamente de sus madres, da lugar a un mecanismo estatal polimórfico en el que sus múltiples formas representan el estado de fuerzas predominantes en un momento determinado.

Por último, focalizar la atención en cómo una repartición estatal plantea el tema del llamado binomio madre-hijo en el transcurso de 19 años habilita la reflexión sobre las diferencias y similitudes existentes en otras latitudes y en los vasos comunicantes entre las políticas sociales surgidas en diferentes países de América. Enfrentar dicha tarea, que excede la problemática de este trabajo, permitiría avanzar sobre la construcción de un modelo teórico metodológico que, sin dejar de considerar aquellos concebidos en Europa y América del Norte, responda a las características particulares del Cono Sur. (Hochman, 2005; Carrillo, 2005; Birn, 2006).

\section{NOTAS}

${ }^{1}$ Ley 12.341: creación de la Dirección de Maternidad e Infancia (Recopilación..., 1938).

${ }^{2}$ Decreto reglamentario 101.341, 11 mar. 1937 (Creación..., jul.-ago. 1937).

${ }^{3}$ Carta del Patronato Nacional de Menores al ministro de Justicia e Instrucción Pública con fecha del 1 de julio de 1937. Archivo General de la Nación, Ministerio del Interior, año 1937, caja 26, legajo 23.871.

${ }^{4}$ Carta del director nacional de Higiene al ministro del Interior del 6 de octubre de 1937. Archivo General de la Nación, Ministerio del Interior, año 1937, caja 26, legajo 23.871.

${ }^{5}$ Opinión del procurador del Tesoro, fechada el 21 de septiembre de 1937. Archivo General de la Nación, Ministerio del Interior, año 1937, caja 26, legajo 23.871.

${ }^{6}$ Opinión del procurador general de la nación, fechada el 20 de octubre de 1937. Archivo General de la Nación, Ministerio del Interior, año 1937, caja 26, legajo 23.871. 
${ }^{7}$ Decreto 5.520, 19 feb. 1938 (Ley de protección..., abr.-jun. 1938).

${ }^{8}$ Diario de sesiones de la Cámara de Diputados, Imprenta del Congreso, p.3869, 1948.

${ }^{9}$ Archivo general de la Nación, Secretaría Técnica, caja 45, iniciativas 10.848 y 15.051 del 21 de diciembre de 1951.

\section{REFERENCIAS}

ARÁOZ ALFARO, Gregorio.

"La protección de la infancia", Boletín del Museo Social Argentino, Buenos Aires, año 16, entregas 67-68, p.19-20. 1928.

\section{ARGENTINA.}

Ministerio de Salud Pública. Memoria correspondiente al período 1946-1952. Buenos Aires: Talleres Gráficos. 1952.

\section{ARGENTINA.}

Ministerio de Salud Pública de la Nación. Política de Salud Pública (1949-1952). Buenos Aires: Talleres Gráficos. 1951a.

\section{ARGENTINA.}

Ministerio de Salud Pública. Plan esquemático de salud pública (1952-1953). Buenos Aires: Ministerio de Salud Pública. 1951b.

\section{ARGENTINA.}

Ministerio de Salud Pública. Reglamento interno: parte técnica general. Buenos Aires:

Departamento de Talleres Gráficos. 1950.

\section{ARGENTINA.}

Secretaría de Salud Pública. Almanaque de Salud. Buenos Aires: Estudio Proteo. 1948.

\section{ARGENTINA.}

Secretaría de Salud Pública. Plan analítico de salud pública. Buenos Aires: s.n. 1947.

\section{ARGENTINA.}

Departamento Nacional de Higiene. Memoria de 1940. Boletín del Departamento Nacional de Higiene, Buenos Aires, p.49-50. 1941.

ARMUS, Diego.

Consenso, conflicto y liderazgo en la lucha contra la tuberculosis. Buenos Aires 1870-1950. In: Suriano, Juan. (Comp.). La cuestión social en Argentina, 1870-1943. Buenos Aires: La Colmena, p.191-216. 2004.

BARRANCOS, Dora.

Iniciativas y debates en materia de reproducción durante el primer peronismo (1946-1952). Salta: Seposal. 2002.

BERUTI, Josué.

Producción científica y cultural. t.3. Buenos Aires: s.n. 1943.

BIERNAT, Carolina.

Médicos, especialistas, políticos y funcionarios en la organización centralizada de la profilaxis de las enfermedades venéreas en la Argentina (1930-1954). Anuario de Estudios Americanos, Sevilla, v.64, n.1, p.257-288. 2007.

BIERNAT, Carolina.

Las dos vías de la imaginación poblacionista argentina: natalismo e inmigración entre 1914 y 1955. Buenos Aires: Ciclos. 2005a.

BIERNAT, Carolina.

La eugenesia argentina y el debate sobre el crecimiento de la población en los años de entreguerras. Cuadernos del Sur (Historia), Bahía Blanca, n.34, p.251-273.2005b.

BILELLA, Roberto.

La mortinatalidad infantil y su organismo de protección en la provincia de San Juan.

Congreso Nacional de Puericultura, 1., 7-10 octubre 1940. Actas y trabajos.... t.2. Buenos Aires: s.n. p.248-265. 1941.

BILLOROU, María José.

La construcción de la puericultura como campo científico y como política pública en Buenos Aires, 1930-1945. Tesis (Maestría) Facultad de Ciencias Humanas/Universidad Nacional de La Pampa, Santa Rosa. 2006.

BIRN, Anne-Emanuelle. O nexo nacional-interacional na saúde pública: o Uruguay e a circulação das políticas e ideologías de saúde infantil, 1890-1940. História, Ciências, Saúde - Manguinhos, Río de Janeiro, v.13. n.3. p.675-708. 2006.

CARrILLO, Ana.

Salud pública y poder en México durante el Cardenismo, 1934-1940. In: Dynamis, v. 25, p.145-178. 2005.

\section{CARRILLO, Ramón.}

La organización del Ministerio de Salud Pública de la Nación. In: Carrillo, Ramón.

Contribuciones al conocimiento sanitario. Buenos Aires: Talleres Gráficos. p.286-304. 1951.

CARRILLO, Ramón.

Los presupuestos de salud pública de 1946 a 1950 en relación con el desarrollo técnico administrativo del organismo nacional. Buenos Aires: Imprenta del Ministerio de Salud Pública. 1950.

CELTON, Dora.

La declinación de la mortalidad en la 
Argentina. In: II Jornadas Argentinas de Población (AEPA). Buenos Aires: Secretaría Parlamentaria. p.325-341. 1995.

\section{CREACIÓN..}

Creación de la Dirección de Maternidad e Infancia. Boletín del Museo Social Argentino, Buenos aires, año 25, entregas 181-182, p.148-166. jul.-ago. 1937.

DEZEO, Pilades. La organización nacional de protección a la maternidad e infancia. Congreso Nacional de Medicina, 6., 16-22 octubre 1938, Córdoba. Actas y trabajos... t.3. S.l.: s.n. p.523-540. 1939.

DI LISCIA, María Herminia et al. Mujeres, maternidad y peronismo. La Pampa: Fondo Editorial Pampeano. 2000.

DI LISCIA, María Silvia.

Hijos sanos y legítimos: sobre matrimonio y asistencia social en Argentina (1935-1948). História, Ciências, Saúde - Manguinhos, Río de Janeiro, v.9, supl., p.209-232. jul.-ago. 2002.

FRIGIERI, Víctor.

La ley de protección a la maternidad y a la infancia: reflexiones de un médico rural. Revista de la Federación Médica de la República

Argentina, Buenos Aires, año 4. n.38, p.25-30. 1944.

GARCÍA, Lorenzo.

Planificación sanitaria. Santa Fe: Universidad Nacional del Litoral. 1954.

GIMÉNEZ LASCANO, Raúl.

La asistencia y protección a la maternidad y la infancia en la provincia de Tucumán. Congreso Nacional de Puericultura, 1., 7-11 octubre 1940. Actas y trabajos.... t.2. Buenos Aires: s.n.. p.189-198. 1941.

GONZÁLEZ LEANDRI, Ricardo.

Madurez y poder: médicos e instituciones sanitarias en la Argentina a fines del siglo XIX. Entrepasados, Buenos Aires, n.27, p.133-150. 2005.

HOCHMAN, Gilberto.

Cambio político y reformas de la salud pública en Brasil: el primer gobierno de Vargas (19301945). Dynamis, Granada, v. 25, p.199-226. 2005.

IPSEN, Carl.

Demografía totalitaria: il problema de la popolazione nell' Italia fascista. Bologna: Il Mulino. 1997.

LEY DE PROTECCIÓN..

Ley de protección maternal e infantil (ley Palacios). Anales de la Sociedad de Puericultura de Buenos Aires, Buenos Aires, t.5, n.2, p.157-172. abr.-jun. 1938.
LOBATO, Mirta.

Entre la protección y la exclusión: discurso maternal y protección de la mujer obrera en Argentina, 1890-1934. In: Suriano, Juan. (Comp.). La cuestión social en la Argentina, 1870-1943. Buenos Aires: La Colmena. p.245-275. 2000.

MAURÍN NAVARRO, Juan.

La tragedia biológica del interior y la función higiénica social de los dispensarios en la lucha contra la mortalidad infantil. Anales de la Sociedad de Puericultura de Buenos Aires, Buenos Aires, t.9, n.1, p.42-68. 1943.

MAZZEO, Victoria.

Mortalidad infantil en la ciudad de Buenos Aires (1856-1986). Buenos Aires: Centro Editor de América Latina. 1993.

MEAD, Karen.

Beneficent maternalism: Argentine matrherhood in comparative perspective, 18801920. Journal of Womens History, Urbana, v.12, n.3, p. 120-145. 2000.

MENCHACA, Francisco.

El organismo básico de la protección sanitaria materno-infantil. Revista de la Sociedad de Puericultura de Buenos Aires, Buenos Aires, t.19, n. 64, p.20-36. 1953.

MENCHACA, Francisco.

El concepto de integración en los planes y construcciones sanitarias. Anales de Medicina Pública, Rosario, n.10, p.120-125. 1952.

MIRANDA, Marisa; VALLEJO, Gustavo (Comp).

Darwinismo social y eugenesia en el mundo latino. Buenos Aires: Siglo XXI de Argentina Editores. 2005.

NARI, Marcela.

Políticas de maternidad y maternalismo político: Buenos Aires, 1890-1940. Buenos Aires: Biblos. 2004.

OLARÁN CHANS, Aníbal.

La legislación argentina en materia de protección a la maternidad y a la infancia. Congreso Nacional de Puericultura, 1., 7-10 octubre 1940. Actas y trabajos.... t.2. Buenos Aires: s.n. p.159-204. 1941.

OLARÁN CHANS, Aníbal.

La obra de la Dirección de Maternidad e Infancia en el interior de la República. Anales de la Sociedad de Puericultura de Buenos Aires, Buenos Aires, t.5, n. 3, p.138-145. 1939.

OLARÁN CHANS, Aníbal; SIRI, Luis. Algunas consideraciones sobre asistencia y protección de la maternidad e infancia en nuestro país. Buenos Aires: s.n. 1929. 
OTERO, Hernán.

La transición demográfica argentina a debate: una perspectiva espacial de las explicaciones ideacionales, económicas y político institucional. In: Otero, Hernán (Dir.). El mosaico argentino: modelos y representaciones del espacio y de la población, siglos XIX y XX. Buenos Aires: Siglo XXI. p.71-170. 2004.

PANTELIDES, Edith.

La transición demográfica Argentina: un modelo no ortodoxo. Desarrollo Económico, Buenos Aires, v.22, n. 88, p.511-534. 1983.

\section{RAMACCIOTTI, Karina.}

Las voces que cuestionaron la política sanitaria del peronismo (1946-1949). In: Suriano, Juan; Lvovich, Daniel (Comp). Las políticas sociales en perspectiva histórica. Buenos Aires: Prometeo/ UNGS. p.169-198. 2006.

RAMACCIOTTI, Karina.

Las huellas de la eugenesia en la política sanitaria. In: Miranda, Marisa; Vallejo, Gustavo (Comp.). Darwinismo social y eugenesia en el mundo latino. Buenos Aires: Siglo XXI ArgentinaEspaña. p.311-347. 2005a.

RAMACCIOTTI, Karina.

Las trabajadoras en la mira estatal: propuestas de reforma de la Caja de Maternidad (1934-1955). Revista Trabajos y Comunicaciones, La Plata, n.30, p.191-215. 2005b.

\section{RECOPILACIÓN..}

Recopilación de leyes, reglamentaciones, decretos y resoluciones en vigencia, año 1938. t.1. Boletín del Departamento Nacional de Higiene, Buenos Aires, p.340-345. 1938.

REGGIANI, Andrés.

La demografía como ideología: poblacionismo e identidad nacional en Francia. Anuario, Rosario, n.17, p.403-423. 1995-1996.

SIRI, Luis.

Bases para la elaboración del plan general de la protección a la infancia en la República Argentina. Congreso Nacional de Puericultura, 1., 7-10 octubre 1940. Actas y trabajos.... t.2. Buenos Aires: s.n. p.205-225. 1941.

STEPAN, Nancy Leys.

The hour of eugenics: race, gender and nation in Latin America. Ithaca: Cornell University Press. 1991.

SURIANO, Juan.

Introducción: una aproximación a la definición de la cuestión social en Argentina. In: Suriano, Juan (Comp). La cuestión social en Argentina, 1870-1943. Buenos Aires: La Colmena. p.1-29. 2004.

TORRADO, Susana. Historia de la familia en la Argentina moderna (1870-2000). Buenos Aires: Ediciones de La Flor. 2003. 